\title{
Socioeconomics Influences on the Water Competencies of Young Adult Polish Males
}

Authors' contribution:
A) conception and design of the study
B) acquisition of data
C) analysis and interpretation of data
D) manuscript preparation
E) obtaining funding

\author{
Kevin Moran ${ }^{1 \text { A-E }}$, Robert Podstawski ${ }^{1}$ A-E , Stefan \\ Mańkowski $^{1 \mathrm{~A}-\mathrm{E}}$, Dariusz Choszcz ${ }^{1 \text { A-E }}$, Zoran Sarevic $^{2}$ A-E \\ ${ }^{1}$ University of Warmia \& Mazury, Olsztyn, Poland \\ ${ }^{2}$ Health Centre Novi Sad, Serbia
}

ABSTRACT

Despite the popularity of aquatic recreation and its well-promoted prophylactic and therapeutic values, not a lot is known about how socio-cultural background influences the acquisition of water competency. The aim of this study was to assess the influence of socio-economic and environmental factors on the swimming and rescue skills of male university students $(n=521)$ aged 19-20 years residing in the Region of Warmia and Mazury, Poland.

An anonymous questionnaire was used as a diagnostic tool to elicit information on participants' social backgrounds (such as parental income and level of education). Participants provided self-estimates of swimming and rescue competency that included non-stop distance swimming, familiarity with selected swimming strokes, and their estimated rescue ability.

The results suggest that students' self-estimated swimming competency, the distance they could swim non-stop, and the capacity to swim various strokes increased in association with higher levels of educational background of the father, higher levels of monthly income, and increased size of residential agglomeration. The level of rescue skills also improved with increases in the educational background of both parents, the size of residential agglomeration, and the monthly budget. No significant differences were found in rescue competency levels when analyzed by the mothers' educational experience. Levels of both swimming and rescue competencies were lower than those reported in comparable studies.

Socio-economic status (such as a lack of discretionary time and income for families with a lower socio-economic status) and place of residence (such as lack of facilities in small villages and towns) mediated the opportunity to acquire water competencies or gain experience with aquatic activity. Methods for addressing socio-economic barriers to the acquisition of swimming and rescue competency are discussed and recommendations for further research are made.

KEYWORDS water competency, swimming skill, rescue competency, drowning prevention, water safety socio-economic status 


\section{Introduction}

Recreational swimming and other water-related activities are extremely popular forms of leisure time pursuits in most developed countries. The prophylactic and therapeutic values of aquatic activity have been well promoted as part of a healthy lifestyle. In Poland, swimming/aquatic activities are not yet as popular as in highly developed countries, but their popularity is increasing steadily with the launch of modern water sports centers across the country (Podstawski \& Choszcz 2015). In the United States, swimming has been identified as the fourth most popular recreational activity (US Census Bureau 2012). In the UK, swimming remains the top sporting activity, with over 2.5 million regular participants, in spite of a recent decline (Sport England 2015). In New Zealand, recreational swimming was the second most frequently reported activity behind walking in 2014/15 (Sport New Zealand 2015), and two-thirds (65\%) of Aucklanders reported participating in aquatic recreation at a beach (36\%) and at the sea (29\%; Sport New Zealand \& Auckland Council 2015).

However, while recreational swimming is invariably viewed in a positive light, it does have attendant risks (McCool, Moran, Ameratunga \& Robinson 2008). In the European Union, where 70\% of Europeans spend their holidays close to water (Schmidt 2002), drowning is the third highest cause of tourist injury, comprising $36 \%$ of fatalities and with an injury risk rate 15 times greater for tourists than for residents (Bauer, Körmer, \& Sector 2005). Much aquatic activity, whether at home or abroad, takes place at open water sites such as beaches, rivers, and lakes - locations that are often unsupervised and unregulated; therefore, the risk of drowning is omnipresent. In Poland, most drowning fatalities occur in open water, either when the victim is in the water ( $60 \%$ of all fatalities) or as a consequence of falling in (18\% of all fatalities; Lin, Wang, Lu, \& Kawach 2015). Furthermore, drowning statistics indicate that young Polish adults aged 15-25 years are a highrisk group, with an annual drowning rate of 2.2 per 100,000, a ranking of 34 out of 60 countries with comparable drowning data (Lin et al. 2015). In 2000-2012, 12,702 people drowned in Poland, and the highest risk of fatal drowning was noted among males aged 45-59, mostly with basic vocational education. In the analyzed period, the drowning mortality rate was highest in the Region of Warmia and Mazury (median of 4.7 per population of 100,000; Halik, Poznańska, Seroka, \& Wojtyniak 2014). This places a premium on the capacity of participants to cope with the risk of drowning through their personal water competency - not only for their own safety, but for that of others as well. In spite of the popularity of aquatic recreation, not a lot is known about how the socio-economic and environmental factors in a person's background influence the skills and knowledge base that informs their understanding of water safety.

Swimming and rescue skills have been cited as critical elements of drowning prevention (Moran 2008; McCool et al. 2008; Berukoff \& Hill 2010; Waller \& Norwood 2011), yet access to those skills varies considerably even among and within high income countries. In the US, black children aged 6-16 years drown almost three times more often than white children (Irwin, Drayer, Irwin, \& Southall 2008). In a study on swimming competency among African American and Hispanic children, almost $60 \%$ of children could not swim, twice the rate of non-swimmers among white children (Waller \& Norwood 2011). Poor swimming capacity was also reported among African and Latino Americans, who are rarely active in water sports such as swimming, diving, water polo, rowing, and sailing at the scholastic, collegiate, and professional levels (Hodge, Harrison, Burden, \& Dixon 2008). Lower levels of aquatic activity and lower levels of swimming and rescue competency have also been reported among New Zealand students of low socio-economic status (Moran 2008) and among those of recent residency (Moran 2007; Moran \& Willcox 2013). A deprivation index used to analyze leisure time among adults in New Zealand found that fewer adults of low socio-economic status engaged in weekly recreational activity compared with those of high socio-economic status (low socioeconomic status, 70\%; high socio-economic status, 77\%; Sports New Zealand 2015). Similarly, people living in the city of Auckland, New Zealand from low deprivation (high socio-economic status) areas took part in more activities than those living in high deprivation (low socio-economic status) areas (4.7 activities versus 3.7 activities; Sport New Zealand and Auckland Council 2015). In the UK, a decline in the proportion of people from lower socio-economic groups participating in sport each week was attributed to a decline in swimming activity, despite the fact that it was the most popular activity (Sport England 2015). 
Socio-economic factors such as income, parents' educational background, and urbanization status have been identified as particularly important in human development (Eiben \& Mascie-Taylor 2004; Eiben, Barabás, Kontra \& Pantó 1996). Arguably, urbanization is one of the major socio-economic factors that has an impact on development (Eveleth \& Tanner 1990). A person's physical development can be influenced by place of residence, for example, a large or small settlement or rural or urban environment (Komlos 1994; Eiben \& Mascie-Taylor 2004). The influence of the material status of a family has been reported in terms of income levels (Roche \& Sun 2003). Parental educational backgrounds have also been identified as influencing participation in various physical activities (Cóté 1999). Swimming participation often depends on the availability of instructional and competitive programs, although even where swimming facilities are available, social exclusivity can sometimes deter individuals from swimming (Hastings, Zahran, \& Cable 2006; Ross, Irwin, Irwin, Martin, \& Ryan 2014). A combination of lack of access to swimming facilities and racial segregation has been identified as a barrier to acquiring swimming skills for black children in the US (Houlihan 2008).

University students have been subject to some enquiry from a public health perspective in Poland (Miazek, Chrzanowska, \& Miazek 2005; Podstawski, Górnik \& Gizińska 2013; Podstawski et al. 2014) and in other countries (Steptoe et al. 2002; Haase, Steptoe, Phil, Sallis, \& Wardle 2006). Leisure time activities on the beach (Podstawski, Klimczak, Kolankowska, et al. 2013; Podstawski, Kolankowska, Gizińska, et al. 2013) and in a swimming pool (Podstawski \& Choszcz 2015) have also been subject to some scrutiny. While some studies have explored swimming and other water competencies among high school students (Berukoff \& Hill 2010; Moran 2006, 2007, 2008, 2010) and university students (Moran et al. 2012; Petrass, Blitvich, McElroy, Harvey \& Moran 2012; Podstawski, Boraczyńska, Skibniewska \& Kolankowska 2013; Podstawski, Klimczak \& Kolankowska 2013; Podstawski, Kolankowska \& Gizińska 2013; Podstawski, Choszcz, Klimczak, Kolankowska \& Żurek 2014; Podstawski, Mańkowski \& Choszcz 2016), the influence of socio-economic and environmental factors has not been extensively researched. A study of Polish female students revealed that socio-economic factors such as higher levels of urbanization and a higher monthly budget positively influenced the swimming skills of women. The remaining factors (i.e., parents' educational background, distance between the place of residence and the nearest lake, proximity of lake beaches) were completely unrelated to swimming skill metrics (Podstawski, Gizińska \& Kolankowska 2014). Some studies have reported on rescue competency among adult populations (Moran \& Stanley 2013; Pearn \& Franklin 2009) and youth (Mora 2008), but none have studied the rescue capacity of young adults. Perceptions of rescue competency and confidence among high school students $(N=2202)$ were analyzed by Moran $(2008)$, who found that most students $(60 \%)$ reported having either no rescue skills (35\%) or poor skills $(25 \%)$. There are no studies presenting the influence of environmental factors on the swimming and rescue skills of university male students in Polish literature.

The aim of the research was to measure the impact of selected socio-economic and environmental factors (as independent variables) on the level of swimming and rescue competencies (as dependent variables) among first-year male university students.

\section{Methods}

\section{Study design}

The study employed a cross-sectional design using survey methodology to elicit information on the socio-economic influences thought likely to influence student water competency. The research was conducted during compulsory physical education classes at the beginning of the summer term in 2015 at the University of Warmia and Mazury (UWM) in Olsztyn, Poland. The study was a complementary part of a study on the healthy lifestyles and motor fitness of students starting their education at UWM and was conducted as part of the "Health - Conscious University" program (Podstawski 2006). The study was approved by the Bioethics Committee of the University of Warmia and Mazury. The male students who volunteered to participate in the research were obliged to sign a written statement of informed consent. The study was conducted in accordance with the World Medical Association (WMA) Declaration of Helsinki. 


\section{Participants}

The research participants consisted of 521 male students enrolled at UWM in their first year of study. The average age of the participants was $19.73 \pm 0.58$ years. The participants were randomly selected from the university's first-year enrollment and then interviewed. The students were informed of the aim of the research during their first physical education class (an organization-regulation meeting). On the basis of the information obtained, only students who were permanent residents of the Region of Warmia and Mazury were selected to take part in the study.

\section{Research Instrument}

The students were surveyed with the use of an anonymous self-complete questionnaire that included 9 closed questions and was designed to be completed in 5-10 minutes. Several questions focused on the subjective assessment of their swimming and rescue competencies. The first question asked them to categorize their swimming competency using a 4-point scale that included cannot swim, swims poorly, swims well, and swims very well. They were then asked to estimate how far they thought they could swim using a 6-point scale ranging from no distance to more than $1,000 \mathrm{~m}$, and whether or not they could perform a range of swimming strokes (such as the front crawl and breaststroke). Rescue skills were estimated according to a 4-point scale that included no skills, poor skills (first aid), good skills (junior lifeguard), and excellent skills (senior lifeguard). The last part of the questionnaire included personal information about age, gender, parents' educational backgrounds, monthly budget, and place of residence.

Place of permanent residence was defined in terms of site description and size of population. The residential environment types included village or small town $(<20,000$ inhabitants), big town $(20,000-50,000$ inhabitants), small city (50,001-100,000 inhabitants), and big city (>100,000 inhabitants). The level of education attained by both the mother and father was defined in terms of primary school, secondary school, or higher education attendance. A further indication of socio-economic status was obtained from estimates of students' monthly income, expressed in Polish złoty and categorized as <1,000 PLN per month, 1,001-1,500 PLN per month, 1,501-2,000 PLN per month, and >2,000 PLN per month.

\section{Statistical analysis}

To determine whether the size of the sample population could be considered to be representative of first-year male university students aged 19-20 years, residing in the Region of Warmia and Mazury, the following formula (1) was used:

$$
n=\frac{\mu_{\alpha}^{2}}{4 d^{2}}
$$

Where: $d$ - the maximum (acceptable) error of estimation, $\mu_{\alpha}-$ the value from the normal distribution table $N(0.1)$ at the accepted confidence coefficient $1-\alpha$. The authors assumed that for the accepted confidence coefficient $1-\alpha=0.90$ ( $\left.\mu_{\alpha}=1.64\right)$, the average estimation error did not exceed 5\% (Krefft \& Choszcz 2000). Using this formula, it was possible to calculate that the minimum number of young male adults in the sample should be 269. Thus the number surveyed $(n=521)$ was deemed appropriate.

All data was entered into the Statistics PL program Version 10 (Luszniewicz \& Słaby 2008) for statistical analysis. Frequency tables using numbers and percentages were generated to report on self-estimated swimming and rescue competencies. For binary data, chi-square tests were used to determine associations among dependent variables (such as swimming and rescue competency) and independent variables (such as parents' level of educational attainment and monthly income). To test the relationship between perceived swimming/rescue competency and socio-economic status via the variables reported above, statistical significance was set as $\alpha=0.05$. In cases where the probability of exceeding the estimated value was lower than the assumed statistical significance $(p<\alpha)$, the differences between the analyzed groups were regarded as significant (Rabiej 2012). 


\section{Results}

Most students (62\%) who took part in the study had been raised in small towns (38\%) or villages (24\%), while the remainder lived in big towns (21\%) and large (13\%) or small cities $(5 \%)$. In terms of parental education, approximately half of the participants' mothers (52\%) and fathers $(47 \%)$ had completed secondary school education. A slightly lesser proportion of mothers $(39 \%)$ and fathers $(42 \%)$ had received higher education. A small proportion of mothers $(9 \%)$ and fathers $(11 \%)$ had received only primary school education. The monthly income of half (53\%) of the male students was less than 1,000 PLN (313 USD), and one-third (34\%) of the students received 1,001-1,500 PLN (313-469 USD) per month. A small proportion (8\%) reported a monthly income of between 1,501 PLN-2,000 PLN (469-625 USD), and only 5\% of the men's monthly income exceeded 2,000 PLN (625 USD).

Most students (88\%) self-reported that they either could not swim $(63 \%)$ or could only swim poorly $(25 \%)$. Only a small proportion (12\%) thought that they could swim well $(7 \%)$ or very well $(5 \%)$. When asked to estimate the distance they could swim without stopping, most (83\%) estimated that they could swim less than $50 \mathrm{~m}$; a further $10 \%$ thought they could swim up to $500 \mathrm{~m}$, and a small proportion (2\%) estimated that they could swim $1 \mathrm{~km}$. Of the half (48\%) who reported that they could swim strokes, breaststroke (19\%) was the most frequently reported, followed by front crawl (10\%), freestyle (9\%), backstroke (8\%), and butterfly $(3 \%)$.

\section{Fathers' level of education}

When student swimming competency was analyzed by the educational background of their fathers, considerable differences were evident in all three estimates of competency (Table 1).

Table 1. Influence of fathers' educational background on swimming skills

\begin{tabular}{|c|c|c|c|c|c|c|c|c|}
\hline \multicolumn{9}{|c|}{ Influence of fathers' educational background on swimming ability } \\
\hline \multirow[t]{2}{*}{ Criteria } & \multicolumn{2}{|c|}{ Primary school } & \multicolumn{2}{|c|}{$\begin{array}{l}\text { Secondary } \\
\text { school }\end{array}$} & \multicolumn{2}{|c|}{$\begin{array}{c}\text { Higher } \\
\text { education }\end{array}$} & \multicolumn{2}{|c|}{ Total } \\
\hline & $\mathbf{N}$ & $\%$ & $\mathbf{N}$ & $\%$ & $\mathbf{N}$ & $\%$ & $\mathbf{N}$ & $\%$ \\
\hline Cannot swim & 45 & 77.6 & 186 & 75.3 & 99 & 45.8 & 330 & 63.3 \\
\hline Swims poorly & 12 & 20.7 & 56 & 22.7 & 62 & 28.7 & 130 & 25.0 \\
\hline Swims well & 1 & 1.7 & 5 & 2.0 & 28 & 13.0 & 34 & 6.5 \\
\hline Swims very well & 0 & 0.0 & 0 & 0.0 & 27 & 12.5 & 27 & 5.2 \\
\hline Total & 58 & 100.0 & 247 & 100.0 & 216 & 100.0 & 521 & 100.0 \\
\hline \multicolumn{9}{|c|}{ Influence of fathers' educational background on estimated swimmable distance [m] } \\
\hline $0 \mathrm{~m}$ & 45 & 77.6 & 186 & 75.3 & 99 & 45.8 & 330 & 63.3 \\
\hline$\leq 50 \mathrm{~m}$ & 11 & 19.0 & 45 & 18.2 & 48 & 22.2 & 104 & 20.0 \\
\hline $51-100 \mathrm{~m}$ & 1 & 1.7 & 11 & 4.5 & 17 & 7.9 & 29 & 5.6 \\
\hline $101-500 \mathrm{~m}$ & 1 & 1.7 & 4 & 1.6 & 20 & 9.3 & 25 & 4.8 \\
\hline $501-1,000 \mathrm{~m}$ & 0 & 0.0 & 1 & 0.4 & 23 & 10.7 & 24 & 4.6 \\
\hline$>1,000 \mathrm{~m}$ & 0 & 0.0 & 0 & 0.0 & 9 & 4.2 & 9 & 1.7 \\
\hline Total & 58 & 100.0 & 247 & 100.0 & 216 & 100.0 & 521 & 100.0 \\
\hline $\begin{array}{c}\text { Mean (meters per } \\
\text { person) }\end{array}$ & \multicolumn{2}{|c|}{11.21} & \multicolumn{2}{|c|}{15.79} & \multicolumn{2}{|c|}{160.76} & \multicolumn{2}{|c|}{75.38} \\
\hline \multicolumn{9}{|c|}{ Influence of fathers' educational background on ability to swim given strokes* } \\
\hline Cannot swim & 45 & 77.6 & 186 & 73.2 & 99 & 30.1 & 330 & 51.5 \\
\hline Front crawl & 0 & 0.0 & 8 & 3.2 & 55 & 16.7 & 63 & 9.8 \\
\hline Butterfly & 0 & 0.0 & 0 & 0.0 & 22 & 6.7 & 22 & 3.4 \\
\hline Backstroke & 0 & 0.0 & 4 & 1.6 & 45 & 13.7 & 49 & 7.6 \\
\hline Breaststroke & 3 & 5.2 & 30 & 11.8 & 86 & 26.1 & 119 & 18.6 \\
\hline Freestyle & 10 & 17.2 & 26 & 10.2 & 22 & 6.7 & 58 & 9.1 \\
\hline Total & 58 & 100.0 & 254 & 100.0 & 329 & 100.0 & 641 & 100.0 \\
\hline
\end{tabular}


Abbreviations: $\mathrm{N}$ - number of responses, $\%$ - percentage, ${ }^{*}$ - the respondents were able to provide multiple answers, primary school and vocational secondary school.

Source: own study

Most students whose fathers had received only primary education $(n=58)$ reported that they could not swim $(n=45,78 \%)$ or could only swim poorly $(n=12,21 \%)$. Of those students whose fathers had undergone secondary education, most reported that they either could not swim $(n=186,75 \%)$ or had poor swimming skills $(n=56,23 \%)$. Students whose fathers had received higher education $(n=216)$ were less likely to be unable to swim (46\%) or to swim poorly (29\%) and were more likely to report that they could swim well (13\%) or very well (13\%). Significant differences were evident in student estimates of swimming competency $(\chi 2(6)$ $=81.6057, p=<0.001)$, estimated swimming distance $(\chi 2(10)=80.2605, p=<0.05)$, and swimming stroke competency $(\chi 2(10)=175.3089, p=<0.001)$ when analyzed by their fathers' level of education.

When asked to estimate swimming competency in terms of the distance they could swim, a similar pattern was evident. Table 1 shows that most students whose father had only received primary education could not swim any distance (78\%), one-fifth thought they could swim up to $50 \mathrm{~m}(19 \%)$, and a small proportion $(<4 \%)$ thought they could swim 51-500 m. Similar estimates of poor swimming ability in terms of distance were also evident among students whose fathers had only attended secondary school $(75.3 \%$ could not swim, $18 \%$ could swim $\leq 50 \mathrm{~m}$ ). For those whose fathers had received higher education, almost half $(46 \%)$ reported that they could not swim any distance, but this group was more likely to report being able to swim more than $50 \mathrm{~m}$ (higher education, 32\%; secondary schooling, 7\%; primary education, $3 \%$ ).

The pattern of lower levels of swimming competency for those whose fathers had lower levels of educational attainment was again evident when swimming stroke competency was evaluated. Table 1 shows that most children of fathers with only primary $(78 \%)$ or secondary education $(73 \%)$ could not swim any stroke. Of the students whose fathers had received higher education, one-third (30\%) could not swim any stroke.

\section{Mothers' level of education}

When analyzed by the level of education attained by the students' mothers, considerable differences were evident in the three estimates of students' swimming competency (Table 2). Statistically significant differences were evident in student estimates of swimming competency $(\chi 2(6)=70.2227, p=<0.001)$, ability to swim an estimated distance $(\chi 2(10)=73.2315, p=<0.05)$, and swimming stroke competency $(\chi 2(10)=$ $112.3980, p=<0.001)$ when analyzed by their mothers' education levels.

Of students whose mothers had received only primary education, most (81\%) could not swim, and only a small proportion $(6 \%)$ could swim well. Of those whose mothers had attained secondary education, threequarters (74\%) could not swim, and very few (4\%) could swim well/very well. Of those whose mothers had received higher education, almost half (45\%) could not swim, but more could swim well/very well (23\%).

When asked to estimate how far they could swim, students whose mothers had received only primary education $(81 \%)$ or secondary schooling $(75 \%)$ were most likely to be unable to swim any distance. Those whose mothers had received higher education were less likely to be unable to swim any distance (45\%) and more likely to be able to swim more than $50 \mathrm{~m}(31 \%)$ when compared with others (primary schooling only, $6 \%$; secondary schooling only, $8 \%)$. More than two-thirds of students $(69 \%)$ whose mothers had received only primary or secondary education could not swim any stroke. Less than one-third (31\%) of students whose mothers had received higher education could not swim any stroke. 
Table 2. Influence of mothers' educational background on swimming skills

\begin{tabular}{|c|c|c|c|c|c|c|c|c|}
\hline \multicolumn{9}{|c|}{ Influence of mothers' educational background on swimming ability } \\
\hline \multirow{2}{*}{ Criteria } & \multicolumn{2}{|c|}{ Primary school } & \multicolumn{2}{|c|}{$\begin{array}{l}\text { Secondary } \\
\text { school }\end{array}$} & \multicolumn{2}{|c|}{ Higher education } & \multicolumn{2}{|c|}{ Total } \\
\hline & $\mathbf{N}$ & $\%$ & $\mathbf{N}$ & $\%$ & $\mathbf{N}$ & $\%$ & $\mathrm{~N}$ & $\%$ \\
\hline Cannot swim & 38 & 80.8 & 200 & 74.3 & 92 & 44.9 & 330 & 63.3 \\
\hline Swims poorly & 6 & 12.8 & 59 & 21.9 & 65 & 31.7 & 130 & 24.9 \\
\hline Swims well & 3 & 6.4 & 8 & 3.0 & 23 & 11.2 & 34 & 6.5 \\
\hline Swims very well & 0 & 0.0 & 2 & 0.7 & 25 & 12.2 & 27 & 5.2 \\
\hline Total & 47 & 100.0 & 269 & 100.0 & 205 & 100.0 & 521 & 100.0 \\
\hline \multicolumn{9}{|c|}{ Influence of mothers' educational background on estimated swimmable distance [m] } \\
\hline $0 \mathrm{~m}$ & 38 & 80.8 & 200 & 74.3 & 92 & 44.9 & 330 & 63.3 \\
\hline$\leq 50 \mathrm{~m}$ & 6 & 12.8 & 48 & 17.8 & 50 & 24.4 & 104 & 20.0 \\
\hline $51-100 \mathrm{~m}$ & 0 & 0.0 & 11 & 4.1 & 18 & 8.8 & 29 & 5.6 \\
\hline $101-500 \mathrm{~m}$ & 1 & 2.1 & 0 & 0.0 & 24 & 11.7 & 25 & 4.8 \\
\hline $501-1,000 \mathrm{~m}$ & 2 & 4.3 & 8 & 3.0 & 14 & 6.8 & 24 & 4.6 \\
\hline$>1,000 \mathrm{~m}$ & 0 & 0.0 & 2 & 0.7 & 7 & 3.4 & 9 & 1.7 \\
\hline Total & 47 & 100.0 & 269 & 100.0 & 205 & 100.0 & 521 & 100.0 \\
\hline $\begin{array}{c}\text { Mean (meters per } \\
\text { person) }\end{array}$ & \multicolumn{2}{|c|}{41.5} & \multicolumn{2}{|c|}{37.3} & \multicolumn{2}{|c|}{133.2} & \multicolumn{2}{|c|}{75.4} \\
\hline \multicolumn{9}{|c|}{ Influence of mothers' educational background on ability to swim given strokes* } \\
\hline Cannot swim & 38 & 70.0 & 200 & 69.0 & 92 & 31.1 & 330 & 51.5 \\
\hline Front crawl & 3 & 5.4 & 15 & 5.2 & 45 & 15.2 & 63 & 9.8 \\
\hline Butterfly & 2 & 3.6 & 4 & 1.4 & 16 & 5.4 & 22 & 3.4 \\
\hline Backstroke & 3 & 5.4 & 7 & 2.4 & 39 & 13.2 & 49 & 7.6 \\
\hline Breaststroke & 8 & 14.5 & 33 & 11.4 & 78 & 26.3 & 119 & 18.6 \\
\hline Freestyle & 1 & 1.8 & 31 & 10.7 & 26 & 8.8 & 58 & 9.0 \\
\hline Total & 55 & 100.0 & 290 & 100.0 & 296 & 100.0 & 641 & 100.0 \\
\hline
\end{tabular}

Abbreviations: $\mathrm{N}$ - number of responses, \% - percentage, ${ }^{*}-$ the respondents were able to provide multiple answers, primary school and vocational secondary school, $\mathrm{p}>0.05$ signifies the lack of significant differences (no influence).

Source: own study

\section{Place of permanent residence}

When student swimming competency was analyzed by place of permanent residence, considerable differences were evident in all three estimates of swimming competency (Table 3). Most students from villages $(n=127)$ reported that they could not swim $(n=105,83 \%)$ or could only swim poorly $(n=22,17 \%)$. Of those raised in small towns, most reported that they either could not swim $(n=156,80 \%)$ or had poor swimming skills ( $n=37,19 \%$ ), while students from larger communities were less likely to be unable to swim (large town, $56 \%$; small city, $36 \%$; large city, $5 \%$ ) or to swim poorly (29\%). City dwellers were more likely to report that they could swim well/very well (small city, 24\%; large city, 73\%). Significant statistical differences were evident in student estimates of swimming competency $(\chi 2(12)=292.6181, p=<0.001)$, estimated swimming distance $(\chi 2(20)=296.7903, p=<0.001)$, and swimming stroke competency $(\chi 2(20)=305.0979, p=<0.001)$ when analyzed by place of permanent residence. 
Table 3. Influence of place of permanent residence on swimming skills

\begin{tabular}{|c|c|c|c|c|c|c|c|c|c|c|c|c|}
\hline \multirow{3}{*}{ Criteria } & \multicolumn{10}{|c|}{ Influence of place of permanent residence on swimming ability } & & \\
\hline & \multicolumn{2}{|c|}{ Village } & \multicolumn{2}{|c|}{ Small town } & \multicolumn{2}{|c|}{ Big town } & \multicolumn{2}{|c|}{ Small city } & \multicolumn{2}{|c|}{ Big city } & \multicolumn{2}{|c|}{ Total } \\
\hline & $\mathbf{N}$ & $\%$ & $\mathbf{N}$ & $\%$ & $\mathbf{N}$ & $\%$ & $\mathbf{N}$ & $\%$ & $\mathbf{N}$ & $\%$ & $\mathbf{N}$ & $\%$ \\
\hline Cannot swim & 105 & 82.7 & 156 & 79.6 & 57 & 53.3 & 9 & 36.0 & 3 & 4.5 & 330 & 63.3 \\
\hline Swims poorly & 22 & 17.3 & 37 & 18.9 & 46 & 43.0 & 10 & 40.0 & 15 & 22.7 & 130 & 24.9 \\
\hline Swims well & 0 & 0.0 & 3 & 1.5 & 4 & 3.7 & 5 & 20.0 & 22 & 33.3 & 34 & 6.5 \\
\hline Swims very well & 0 & 0.0 & 0 & 0.0 & 0 & 0.0 & 1 & 4.0 & 26 & 39.4 & 27 & 5.2 \\
\hline Total & 127 & 100.0 & 196 & 100.0 & 107 & 100.0 & 25 & 100.0 & 66 & 100.0 & 521 & 100.0 \\
\hline \multicolumn{13}{|c|}{ Influence of place of permanent residence on estimated swimmable distance [m] } \\
\hline $0 \mathrm{~m}$ & 105 & 82.7 & 156 & 79.6 & 57 & 53.3 & 9 & 36.0 & 3 & 4.5 & 330 & 63.3 \\
\hline$\leq 50 \mathrm{~m}$ & 20 & 15.7 & 31 & 15.8 & 37 & 34.6 & 10 & 40.0 & 6 & 9.1 & 104 & 20.0 \\
\hline $51-100 \mathrm{~m}$ & 2 & 1.6 & 7 & 3.6 & 9 & 8.4 & 0 & 0.0 & 11 & 16.7 & 29 & 5.6 \\
\hline $101-500 \mathrm{~m}$ & 0 & 0.0 & 1 & 0.5 & 2 & 1.9 & 4 & 16.0 & 18 & 27.3 & 25 & 4.8 \\
\hline $501-1,000 \mathrm{~m}$ & 0 & 0.0 & 1 & 0.5 & 2 & 1.9 & 1 & 4.0 & 20 & 30.3 & 24 & 4.6 \\
\hline$>1,000 \mathrm{~m}$ & 0 & 0.0 & 0 & 0.0 & 0 & 0.0 & 1 & 4.0 & 8 & 12.1 & 9 & 1.7 \\
\hline Total & 127 & 100.0 & 196 & 100.0 & 107 & 100.0 & 25 & 100.0 & 66 & 100.0 & 521 & 100.0 \\
\hline $\begin{array}{l}\text { Mean (meters } \\
\text { per person) }\end{array}$ & \multicolumn{2}{|c|}{5.1} & \multicolumn{2}{|c|}{12.0} & \multicolumn{2}{|c|}{34.6} & \multicolumn{2}{|c|}{128} & \multicolumn{2}{|c|}{445.1} & \multicolumn{2}{|c|}{75.4} \\
\hline \multicolumn{13}{|c|}{ Influence of place of permanent residence on ability to swim given strokes* } \\
\hline Cannot swim & 105 & 82.7 & 156 & 78.4 & 57 & 51.8 & 9 & 27.3 & 3 & 1.7 & 330 & 51.5 \\
\hline Front crawl & 0 & 0.0 & 6 & 3.0 & 6 & 5.4 & 4 & 12.1 & 47 & 27.3 & 63 & 9.8 \\
\hline Butterfly & 0 & 0.0 & 0 & 0.0 & 0 & 0.0 & 2 & 6.1 & 20 & 11.6 & 22 & 3.4 \\
\hline Backstroke & 4 & 3.1 & 1 & 0.5 & 0 & 0.0 & 2 & 6.1 & 42 & 24.4 & 49 & 7.6 \\
\hline \multirow{2}{*}{$\begin{array}{c}\text { Breaststroke } \\
\text { Freestyle }\end{array}$} & 8 & 6.3 & 15 & 7.5 & 26 & 23.6 & 12 & 36.4 & 58 & 33.7 & 119 & 18.6 \\
\hline & 10 & 7.9 & 21 & 10.5 & 21 & 19.1 & 4 & 12.1 & 2 & 1.2 & 58 & 9.0 \\
\hline Total & 127 & 100.0 & 199 & 100.0 & 110 & 100.0 & 33 & 100.0 & 172 & 100.0 & 641 & 100.0 \\
\hline
\end{tabular}

Abbreviations: $\mathrm{N}$ - number of responses, \% - percentage, * - the respondents were able to provide multiple answers, primary school and vocational secondary school.

Source: own study

Table 3 shows that when students were asked to estimate the distance they thought they could swim, students from smaller villages and towns were most likely to report being unable to swim any distance (villages, 83\%; small towns, 80\%). Those who lived in larger towns and cities were less likely to report being unable to swim any distance and more likely to report being able to swim more than $50 \mathrm{~m}$ (large town, 10\%; small city, 24\%; large city, 86\%). The pattern of lesser competency for those from smaller villages and small towns was also evident when stroke competency was evaluated. Table 3 shows that more village or small town dwellers were unlikely to be able to swim different strokes (villages, $83 \%$; small towns, $78 \%$ ).

\section{Monthly income}

When student swimming competency was analyzed by monthly income, considerable differences were evident in all three estimates of swimming competency (Table 4). Most students with low monthly incomes $(n$ $=275)$ reported that they could not swim $(n=261,95 \%)$. Most students with high monthly incomes $(\mathrm{n}=28)$ reported that they could swim well $(n=10,36 \%)$ or very well $(n=17,61 \%)$. Students with low monthly incomes were also more likely to report being unable to swim any distance $(95 \%)$ or any stroke $(95 \%)$. Students with high monthly incomes were more likely to report being able to swim greater distances ( $>500 \mathrm{~m}, 61 \%)$ and more strokes. Significant statistical differences were evident in student estimates of swimming competency $(\chi 2(9)=398.4869, p=<0.001)$, estimated swimming distance $(\chi 2(15)=154.7850, p=<0.001)$, and swimming stroke competency $(\chi 2(15)=262.7427, p=<0.001)$ when analyzed by monthly income. 
Table 4. Influence of monthly budget (PLN) on swimming skills

\begin{tabular}{|c|c|c|c|c|c|c|c|c|c|c|}
\hline \multicolumn{11}{|c|}{ Influence of monthly budget on swimming ability } \\
\hline \multirow{2}{*}{ Criteria } & \multicolumn{2}{|c|}{$\leq 1,000$} & \multicolumn{2}{|c|}{$1,001-1,500$} & \multicolumn{2}{|c|}{$1,501-2,000$} & \multicolumn{2}{|c|}{$<\mathbf{2 , 0 0 0}$} & \multicolumn{2}{|c|}{ Total } \\
\hline & $\mathbf{N}$ & $\%$ & $\mathbf{N}$ & $\%$ & $\mathbf{N}$ & $\%$ & $\mathbf{N}$ & $\%$ & $\mathbf{N}$ & $\%$ \\
\hline Cannot swim & 261 & 94.9 & 69 & 39.4 & 0 & 0.00 & 0 & 0.0 & 330 & 63.3 \\
\hline Swims poorly & 14 & 5.1 & 103 & 58.9 & 12 & 27.9 & 1 & 3.6 & 130 & 24.9 \\
\hline Swims well & 0 & 0.0 & 3 & 1.7 & 21 & 48.8 & 10 & 35.7 & 34 & 6.5 \\
\hline Swims very well & 0 & 0.0 & 0 & 0.00 & 10 & 23.3 & 17 & 60.7 & 27 & 5.2 \\
\hline Total & 275 & 100.0 & 175 & 100.0 & 43 & 100.0 & 28 & 100.0 & 521 & 100.0 \\
\hline \multicolumn{11}{|c|}{ Influence of monthly budget on estimated swimmable distance [m] } \\
\hline $0 \mathrm{~m}$ & 261 & 94.9 & 69 & 39.4 & 0 & 0.0 & 0 & 0.0 & 330 & 63.3 \\
\hline$\leq 50 \mathrm{~m}$ & 13 & 4.7 & 80 & 45.7 & 11 & 25.6 & 0 & 0.0 & 104 & 20.0 \\
\hline $51-100 \mathrm{~m}$ & 1 & 0.4 & 20 & 11.4 & 7 & 16.3 & 1 & 3.6 & 29 & 5.6 \\
\hline $101-500 \mathrm{~m}$ & 0 & 0.0 & 4 & 2.3 & 11 & 25.6 & 10 & 35.71 & 25 & 4.8 \\
\hline $501-1,000 \mathrm{~m}$ & 0 & 0.0 & 2 & 1.1 & 10 & 23.3 & 12 & 42.9 & 24 & 4.6 \\
\hline$>1,000 \mathrm{~m}$ & 0 & 0.0 & 0 & 0.0 & 4 & 9.3 & 5 & 17.9 & 9 & 1.7 \\
\hline Total & 275 & 100.0 & 175 & 100.0 & 43 & 100.0 & 28 & 100.0 & 521 & 100.0 \\
\hline $\begin{array}{c}\text { Mean (meters per } \\
\text { person) }\end{array}$ & \multicolumn{2}{|c|}{10.0} & \multicolumn{2}{|c|}{35.4} & \multicolumn{2}{|c|}{362.8} & \multicolumn{2}{|c|}{609.8} & \multicolumn{2}{|c|}{75.4} \\
\hline \multicolumn{11}{|c|}{ Influence of monthly budget on ability to swim given strokes* } \\
\hline Cannot swim & 261 & 94.9 & 69 & 37.9 & 0 & 0.0 & 0 & 0.0 & 330 & 51.5 \\
\hline Front crawl & 0 & 0.0 & 9 & 4.9 & 26 & 26.8 & 28 & 32.2 & 63 & 9.8 \\
\hline Butterfly & 0 & 0.0 & 0 & 0.0 & 8 & 8.2 & 14 & 16.1 & 22 & 3.4 \\
\hline Backstroke & 0 & 0.0 & 9 & 4.9 & 20 & 20.6 & 20 & 23.0 & 49 & 7.6 \\
\hline Breaststroke & 6 & 2.2 & 53 & 29.1 & 35 & 36.1 & 25 & 28.7 & 119 & 18.6 \\
\hline Freestyle & 8 & 2.9 & 42 & 23.1 & 8 & 8.2 & 0 & 0.0 & 58 & 9.0 \\
\hline Total & 275 & 100.0 & 182 & 100.0 & 97 & 100.0 & 87 & 100.0 & 641 & 100.0 \\
\hline
\end{tabular}

Abbreviations: $\mathrm{N}$ - number of responses, $\%$ - percentage, ${ }^{*}-$ the respondents were able to provide multiple answers.

Source: own study

\section{Rescue skills}

When student rescue competency was analyzed by their fathers' educational background, place of residence, and monthly income, considerable differences were evident in all three estimates of swimming competency (Table 5). Statistically significant differences were evident when rescue competency was analyzed by students' fathers' educational background $(\chi 2(9)=398.4869, p=<0.001)$. Of the students whose fathers had only primary or secondary education, almost all (98\%) reported that they had no rescue skills. Students whose fathers had received higher education were more likely to possess some rescue skills (24\%). Of the students whose mothers had received only primary education or secondary education, almost all of them (94\%) claimed that they had no rescue competencies. Although more students whose mothers had received higher education reported some rescue competency (20\%), the difference was not statistically significant (Table 5).

When analyzed by place of permanent residence, no village-dwelling students reported any rescue competency. In contrast, city-dwelling students were more likely to report having rescue skills (small cites, 24\%; large cities, 73\%). Statistically significant differences were evident when rescue competency was analyzed by place of permanent residence $\left(\chi^{2}(15)=154.7850, p=<0.001\right)$. When rescue competency was analyzed by monthly income, students with low monthly incomes were least likely to possess rescue competencies, while those with high monthly incomes were most likely to possess rescue capacity (lowest monthly income, $0 \%$ rescue capacity; highest monthly income, $75 \%$ good/very good skills). Table 5 also shows a statistically significant difference when rescue competency was analyzed by monthly income $(\chi 2(15)=$ 262.7427, $p=<0.001)$. 
Table 5. Influence of fathers' and mothers' educational background, place of residence, and monthly budget (PLN) on the rescue skills of the evaluated students

\begin{tabular}{|c|c|c|c|c|c|c|c|c|c|c|c|c|}
\hline \multicolumn{13}{|c|}{ Influence of fathers' educational background on rescue skills } \\
\hline \multirow{2}{*}{ Criteria } & \multicolumn{3}{|c|}{ Primary school } & \multicolumn{3}{|c|}{ Secondary school } & \multicolumn{3}{|c|}{ Higher education } & \multicolumn{3}{|c|}{ Total } \\
\hline & $\mathbf{N}$ & \multicolumn{2}{|c|}{$\%$} & $\mathbf{N}$ & \multicolumn{2}{|c|}{$\%$} & $\mathbf{N}$ & \multicolumn{2}{|c|}{$\%$} & $\mathbf{N}$ & & $\%$ \\
\hline No skills & 57 & \multicolumn{2}{|c|}{98.3} & 242 & \multicolumn{2}{|c|}{98} & 159 & \multicolumn{2}{|c|}{73.6} & 458 & & 87.9 \\
\hline Poor skills & 1 & \multicolumn{2}{|c|}{1.7} & 5 & \multicolumn{2}{|c|}{2.0} & 26 & \multicolumn{2}{|c|}{12.0} & 32 & & 6.1 \\
\hline Good & 0 & \multicolumn{2}{|c|}{0.0} & 0 & \multicolumn{2}{|c|}{0.0} & 17 & \multicolumn{2}{|c|}{7.9} & 17 & & 3.3 \\
\hline Very good & 0 & \multicolumn{2}{|c|}{0.0} & 0 & \multicolumn{2}{|c|}{0.0} & 14 & \multicolumn{2}{|c|}{6.5} & 14 & & 2.7 \\
\hline Total & 58 & \multicolumn{2}{|c|}{100.0} & 247 & \multicolumn{2}{|c|}{100.0} & 216 & \multicolumn{2}{|c|}{100.0} & 521 & & 100.0 \\
\hline \multicolumn{13}{|c|}{ Influence of mothers' educational background on rescue skills } \\
\hline No skills & 44 & \multicolumn{2}{|c|}{93.6} & 249 & \multicolumn{2}{|c|}{92.6} & 165 & \multicolumn{2}{|c|}{80.5} & 458 & & 87.9 \\
\hline Poor skills & 0 & 0. & & 10 & 3.7 & & 22 & 10 & & 32 & & 6.1 \\
\hline Good & 3 & 6. & & 8 & 3. & & 6 & 2. & & 17 & & 3.3 \\
\hline Very good & 0 & 0. & & 2 & 0. & & 12 & 5. & & 14 & & 2.7 \\
\hline Total & 47 & 100 & & 269 & 10 & & 205 & 100 & & 521 & & 100.0 \\
\hline & & Influen & e of 1 & lace of & rman & nt resid & ence on & rescue & kills & & & \\
\hline Criteria & & age & Sm: & ll town & Big & town & Sma & I city & Big ci & & & Total \\
\hline & $\mathbf{N}$ & $\%$ & $\mathbf{N}$ & $\%$ & $\mathbf{N}$ & $\%$ & $\mathbf{N}$ & $\%$ & $\mathbf{N}$ & $\%$ & $\mathbf{N}$ & $\%$ \\
\hline No skills & 127 & 100.0 & 193 & 98.5 & 103 & 96.3 & 19 & 76.0 & 18 & 27.3 & 458 & 87.9 \\
\hline Poor skills & 0 & 0.0 & 2 & 1.0 & 2 & 1.9 & 3 & 12.0 & 23 & 34.8 & 32 & 6.1 \\
\hline Good & 0 & 0.0 & 1 & 0.5 & 2 & 1.9 & 2 & 8.0 & 12 & 18.2 & 17 & 3.3 \\
\hline Very good & 0 & 0.0 & 0 & 0.0 & 0 & 0.0 & 1 & 4.0 & 13 & 19.7 & 14 & 2.7 \\
\hline Total & 127 & 100.0 & 196 & 100.0 & 107 & 100.0 & 25 & 100.0 & 66 & 00.0 & 521 & 100.0 \\
\hline & & & aflue & cee of $\mathrm{m}$ & thly b & Idget $o$ & n rescue & skills & & & & \\
\hline & & 000 & & $1,000-1,5$ & & 1,500 & 2,000 & & 2,000 & & To & tal \\
\hline Criteria & $\mathbf{N}$ & $\%$ & I & & & $\mathbf{N}$ & $\%$ & $\mathbf{N}$ & $\%$ & & $\mathbf{N}$ & $\%$ \\
\hline No skills & 275 & 100.0 & 17 & & & 10 & 23.3 & 1 & 3.6 & & 458 & 87.9 \\
\hline Poor skills & 0 & 0.0 & 3 & & & 23 & 53.5 & 6 & 21.4 & & 32 & 6.1 \\
\hline Good & 0 & 0.0 & $\mathrm{c}$ & & & 7 & 16.3 & 10 & 35.7 & & 17 & 3.3 \\
\hline Very good & 0 & 0.0 & c & & & 3 & 7.0 & 11 & 39.3 & & 14 & 2.7 \\
\hline Total & 275 & 100.0 & 17 & & & 43 & 100.0 & 28 & 100.0 & & 521 & 100.0 \\
\hline
\end{tabular}

Source: own study

\section{Discussion}

In this study of young adult male students enrolled in the first year of a university program, we explored the influence of socio-economic factors on swimming and rescue competencies. We asked participants to report on their estimated swimming competency using three measures of self-estimation: a subjective assessment, the distance they could swim, and stroke competency. In addition, we asked them to categorize their rescue competency using a 4-point self-rating scale. The results suggest that socioeconomic/environmental factors exerted considerable influence on the swimming and rescue competencies of the students who took part in the study.

Overall, self-estimates of swimming competency, the ability to swim a certain distance, stroke performance, and rescue competency do not suggest a high level of water competency among respondents, with almost two-thirds (63\%) reporting that they were unable to swim and almost all (88\%) having no rescue competency. Similarly low levels of swimming competency were reported among female students in Poland, with almost three-quarters (72\%) unable to swim or only able to swim poorly (26\%) (Podstawski, Gizińska \& Kolankowska 2014). Another Polish study found similarly low levels of swimming competency among female 
students, with only $2 \%$ of them able to swim 100 to $1,000 \mathrm{~m}$, and none able to swim more than $1,000 \mathrm{~m}$ (Podstawski, Boraczyńska, Skibniewska \& Kolankowska 2013). While males in the current study appear to have a slightly greater competency than their female counterparts from previous studies, the generally low levels of swimming competency for both sexes is a cause for concern since swimming competency is assumed to protect against drowning.

Comparisons with the findings of similar international studies reinforce this concern. An international study called the "Can You Swim?" Project, which examined first-year university students from New Zealand, Australia, Norway, and Japan $(N=373)$, reported higher estimates of real and perceived swimming competency than the students in the present study, with most students (76\%) able to swim more than $300 \mathrm{~m}$ non-stop, and two-thirds (67\%) able to swim $100 \mathrm{~m}$ using the backstroke (Moran et al. 2012). Given the similarity of sample populations and the research methodology of the current Polish study and the international "Can You Swim?" study reported above, socio-cultural differences in background may help explain the disparities in swimming competencies between countries.

A study of 2,000 high school students in New Zealand showed that only $4 \%$ of high school students aged 16 years or older could not swim at all (Moran 2008). More than one-quarter (27\%) thought that they could swim between 100-400 m, and one-fifth (19\%) thought they could swim more than $400 \mathrm{~m}$. Studies among members of the general public suggest various estimates of swimming competency. A study of 3,000 beachgoers in New Zealand found that one-third of adult males (34\%) and one-quarter of adult females (24\%) reported being able to swim more than $200 \mathrm{~m}$ (McCool, Moran, Ameratunga \& Robinson 2008). A survey of festivalgoers in a multicultural setting reported that more than a third (39\%) of respondents thought they could swim more than $100 \mathrm{~m}$, and only a small proportion $(<7 \%)$ reported not being able to swim at all (Moran \& Stanley 2013). Caution needs to be exercised when using self-reported swimming competency as a measure of protection against drowning. A recent study of parents in New Zealand $(N=306)$ found that most $(58 \%)$ considered themselves to be good/very good swimmers (58\%), but more than half (55\%) estimated that they could only swim up to $25 \mathrm{~m}$, and, furthermore, many (59\%) had not swum their estimated distance in open water (where most drowning occurs), or had not swum that distance within the past year (39\%; Stanley \& Moran, in press).

With the exception of the influence of mothers' educational background on rescue competency, all other socio-cultural factors measured in the study had a significant impact on the male students' level of swimming and rescue competency. Increased levels of swimming competency relative to the higher levels of educational experience of the parents may be related to higher family income, with the male parent being the dominant income earner. It may also be that those from family backgrounds with greater discretionary time and income may have greater opportunities to engage in aquatic activities and be more able to spend money on acquiring water competency. Conversely, those from limited educational backgrounds are likely to have less discretionary spending power or time to engage in aquatic recreation. A similar phenomenon has been observed among black communities (Houlihan 2008; Ross et al. 2014) and Latino and African American communities (Hastings, Zahran \& Cable 2006; Irwin, Drayer, Irwin \& Southall 2008; Irwin, Irwin, Ryan \& Drayer 2009), where the number of swimming pools is decidedly lower than in white communities.

The association between low income levels and low levels of swimming competency evident in the study suggests that socio-economic status is a strong predictor of swimming capacity. The Region of Warmia and Mazury is an area of high unemployment and has one of the lowest per capita income levels in Poland, so the low levels of swimming competency reported among males in this study are perhaps understandable. Similar findings among young female adults from the same region with low socio-economic status and fewer opportunities to participate in aquatic activities have also been previously reported (Podstawski, Gizińska \& Kolankowska 2014). In other studies, lack of funds for swimming lessons has been identified as a limiting factor in accessing swimming facilities or receiving swimming lessons (Hastings et al. 2006; Waller \& Norwood 2011). As a consequence of this inequality, it is suggested that educational programs that focus on the development of swimming competency be promoted in schools and communities that have low socioeconomic status via subsidized private and public swimming and water safety instruction. 
The impact of the students' place of residence on water competency is not only significant but compelling. Those who lived in small settlements such as villages and small towns reported significantly poorer water competency than city dwellers because they were more likely to be isolated from swimming pool facilities. Conversely, those living in cities were more likely to have access to aquatic recreational facilities (indoor swimming pools, aqua parks, spas). The Polish Region of Warmia and Mazury has only acquired such facilities in the last five years. Consequently, low swimming skills reported in this study may reflect the former lack of opportunity for aquatic activity and the acquisition of swimming and rescue skills. Other studies have reported similar findings, leading Berukoff and Hill (2010) to the conclusion that "the swimming performance of older students appears to be strongly related to opportunities to learn to swim as a child, and the availability of swimming pools as a child" (p. 410). In the Region of Warmia and Mazury, three new swimming pools, including two aqua parks, were recently opened in Olsztyn, resulting in greater opportunities to participate in aquatic activities (Podstawski, Kolankowska, Gizińska, Klimczak \& Górnik 2013). Currently, five public swimming pools are available in Olsztyn, as well as swimming pools in private hotels and spa centers in the Region of Warmia and Mazury. This increased availability of swimming pools and the desire to develop aquatic activities, which is now common in Warmia and Mazury (Podstawski, Klimczak, Kolankowska \& Gizińska 2013), may encourage university students in the area to take up swimming in the future. Previous studies have suggested that city-based students have easier access to such facilities and can, consequently, participate in various water activities more often (Podstawski \& Choszcz 2016). The possibility of subsidized transport to facilitate travel to urban-based pools for school students from rural settlements may be one way of addressing this inequity.

Self-reported rescue competency among male students also appears to be lacking with almost all participants $(88 \%)$ who claimed to have no rescue skills. Other studies have reported a similar lack of rescue competencies, even though the self-reported skill levels were higher than those reported in this study. One Australian study found that more than half of fit adults tested in a simulated drowning incident on dry land could not throw a lifeline accurately (Pearn \& Franklin 2009). Other studies have identified a lack of lifesaving training (Gulliver \& Begg 2005; Moran 2008, 2009; Moran \& Stanley 2013). A nationwide water safety survey of youths in New Zealand found that one-third (35\%) claimed that they had no rescue ability, and more than one-half (59\%) expressed doubts about their ability to perform a deep-water rescue (Moran 2008). A lack of rescue ability has also been reported among 21 -year-olds, most of whom $(52 \%)$ had not received any lifesaving training (Gulliver \& Begg 2005). In a study of parents/caregivers $(N=769)$ of children under 10 years of age at 18 beaches in New Zealand, more than three-quarters (76\%) of the adults surveyed had not received any rescue/lifesaving training (Moran 2009).

The pattern of lower water competency among those with fathers who had lower educational attainment, those from small communities, and those with lower income discussed above in relation to swimming competency is repeated with respect to rescue competency. While the overall estimation of rescue competency was low, even students whose parents had received higher education reported having no rescue skills (father, $74 \%$; mother, $81 \%$ ), as did those from villages (100\%), towns (small towns, $98 \%$; large towns, 96\%), and small cities (76\%). In contrast to this, fewer students from large cities (27\%) and on higher monthly incomes $(>1,500$ PLN, $27 \%)$ reported having no rescue skills. Given the higher levels of drowning previously reported among populations with a low socio-economic status (for example, Brenner, Saluja \& Smith 2003) and rural residents (for example, Fralick, Gallinger \& Hwang 2013), it is recommended that targeted educational programs be introduced to all schools in order to develop bystander rescue skills, especially in rural and lowincome areas where the lack of knowledge is likely to be the greatest.

\section{Limitations}

While this study offers new and robust evidence as to how swimming competency is influenced by background socio-cultural factors, the findings should be treated with caution in light of several methodological limitations. First, because the study was cross-sectional in nature, the associations seen in this study cannot be assumed to be causal, but only relational. Further studies with other sub populations are 
required to determine whether the findings are generalizable. Second, even though they are often used in studies of water safety, self-estimations of swimming competency can result in measurement errors and might not accurately express true competence (Mickalide 1997; Robertson 1992). Further research using actual water competencies may shed further light on the relationship between water competency and socio-economic and environmental influences. Third, as the selected population was part of a cohort enrolled in a compulsory physical education module, it is likely that they have greater competency levels than those of the normal population. Further studies with a more representative sample of the normal population are recommended.

\section{Conclusions}

The results of the research show that socio-economic/environmental factors such as place of permanent residence, parents' level of education, and monthly income have a significant influence on the acquisition of swimming and rescue skills among male university students. On the basis of the differing levels of water competency among males found in this study, targeted educational interventions at the school and community levels have been recommended. The economic cost to the country of lives lost to drowning, as well as the subsequent emotional and social costs to families and society, suggest that such an investment is not only worthwhile but essential.

\section{REFERENCES}

Bauer, R., Körmer, C., \& Sector, M. (2005). Scope and patterns of tourist injuries in the European Union. International Journal of Injury Control and Safety Promotion, 12(1), 57-61.

Berukoff, K.D., \& Hill, G.M. (2010). A study of factors that influence the swimming performance of Hispanic high school students. International Journal of Aquatic Research and Education, 4(4), 409-421.

Brenner, R.A., Saluja, G. \& Smith, G.S. (2003). Swimming lessons, swimming ability, and the risk of drowning. International Journal of Injury Control and Safety Promotion, 10(4), 211-216.

Cóté, Q. (1999). The influence of the family in the development of talent in sport. The Sport Psychologist, 13, 395-417.

Eiben, O.G., Barabás, A., Kontra, G., \& Pantó, E. (1996). Differences in growth and physical fitness of Hungarian urban and rural boys and girls. Homo, 47, 191-205.

Eiben, O.G., \& Mascie-Taylor, C.D.N. (2004). Children's growth and socio-economic status in Hungary. Economic and Human Biology, 2, 295-320.

Eveleth, P.B., \& Tanner, J.M. (1990). Worldwide Variation in Human Growth. Cambridge: Cambridge University Press.

Fralick, M., Gallinger, Z.R., \& Hwang, S.W. (2013). Differences in drowning rates between rural and non-rural residents of Ontario, Canada. International Journal of Aquatic Research and Education, 7(4), 332-343.

Gulliver, P. \& Begg, D. (2005). Usual water-related behaviour and 'near-drowning' incidents in young adults. Australian and New Zealand Journal of Public Health, 29(3), 238-243.

Haase, A., Steptoe, A., Phil, D., Sallis, J.F., \& Wardle, J. (2006). Leisure-time activity in university students from 23 countries: Association with health beliefs, risk awareness, and national economic development. Preventive Medicine, 39 , 182-190.

Halik, R., Poznańska, A., Seroka, W., \& Wojtyniak, B. (2014). Wypadkowe utonięcia w Polsce w latach 2000-2012 /Accidents of drowning in Poland in 2000-2012/. Przeglad Epidemiologiczny, 68, 591-594.

Hastings, D.W., Zahran, S., \& Cable, S. (2006). Drowning in inequalities: Swimming and social justice. Journal of Black Studies, 36(6), 894-917.

Hodge, S.R., Harrison, L., Jr., Burden, J.W., \& Dixon, A.D. (2008). Brown in black and white - then and now: A question of educating or sporting African American males in America. The American Behavioral Scientist, 51(7), 928-952.

Houlihan, B. (2008). Sport and Society: A Student Introduction (2 $2^{\text {nd }}$ ed.). Los Angeles, CA: Sage.

Irwin, C., Irwin, R., Ryan, T., \& Drayer, J. (2009). The mythology of swimming: Are myths impacting minority youth participation? International Journal of Aquatic Research and Education, 3(1), 10-23.

Irwin, R., Drayer, J., Irwin, C.R.T., \& Southall, R. (2008). Constraints impacting minority swimming participation. University of Memphis: Department of Health and Sports Sciences. Retrieved March 26, 2016, from http://www.swimfoundation.org/NETCOMMUNITY/Page.aspx.

Komlos, J. (1994). Preface. In J. Komlos (Ed.), Stature, Living Standards, and Economic Development: Essays in Anthropometric History (pp. 9-11). Chicago: The University of Chicago Press. 
Krefft, R., \& Choszcz, D. (2000). Statistics (Selected problems) [in Polish]. Olecko: Wydawnictwo Wszechnicy Mazurskiej.

Lin, C-Y., Wang, Y-F., Lu, T-H., \& Kawach, I. (2015). Unintentional drowning mortality, by age and body of water: An analysis of 60 countries. Injury Prevention, 21(e1), e43-50.

DOI: 10.1136/injuryprev-2013-041110.

Luszniewicz, A., \& Słaby, T. (2008). Statystyka z pakietem komputerowym STATISTICA PL. Teoria i zastosowania, wydanie 3 [Statistics of computer package STATISTICA PL. Theory and applications, third edition. In Polish.]. Gliwice: C.H. Beck Press.

McCool, J.P., Moran, K., Ameratunga, S. \& Robinson, E. (2008). New Zealand beachgoers' swimming behaviours, swimming abilities and perception of drowning risk. International Journal of Aquatic Research and Education, 2(1), 715.

Miazek, U., Chrzanowska, M., \& Miazek, Z. (2005). Physical activity in student lifestyle in the light recommendation of health promotions experts. Annales Universitatis Mariae Curie - Skłodowska Lublin - Polonia, 80(316), 414-418.

Mickalide, A. (1997). Threats to measurement validity in self-reported data can be overcome. Injury Prevention, 3 , 7-8.

Moran, K. (2006). Water safety knowledge, attitudes and behaviours of Asian youth in New Zealand. In S. Tse, M.E. Hoque, K. Rasanathan, M. Chatterji, R. Wee, S. Garg \& Y. Ratnasabapathy (Eds.), Prevention, Protection and Promotion. Proceedings of the Second International Asian Health and Wellbeing Conference (pp.13-14). Auckland, New Zealand: University of Auckland.

Moran, K. (2007). Water safety knowledge, attitudes and behaviours of young Pasifika New Zealanders. New Zealand Journal of Educational Studies, 42(1\&2), 161-169.

Moran, K. (2008). Will they sink or swim? New Zealand youth water-safety knowledge and skills. International Journal of Aquatic Research and Education, 2(1), 113-126.

Moran, K. (2009). Parent/caregiver perceptions and practice of water safety at the beach.

International Journal of Injury Control and Safety Promotion, 16(4), 215-221.

Moran, K. (2010). Risk of drowning: The "Iceberg Phenomenon" revisited. International Journal of Aquatic Research and Education, 4(2), 115-126.

Moran, K., Stallman, R.K., Kjendlie P.-L., Dahl, D., Blitvich, J. D., Petrass, L.A., McElroy, G.K., Goya, T., Teramoto, K., Matsui, A., \& Shimongata, S. (2012). Can you swim? An exploration of measuring real and perceived water competency. International Journal of Aquatic Research and Education, 6(2), 122-135.

Moran, K., \& Stanley, T. (2013). Readiness to rescue: Bystander perceptions of their capacity to respond in a drowning emergency. International Journal of Aquatic Research and Education, 7(4), 290-300.

Moran, K., \& Willcox, S. (2013). Water safety practices and perceptions of "new” New Zealanders. International Journal of Aquatic Research and Education, 7(2), 136-146.

Pearn, J., \& Franklin, R.C. (2009). "Flinging the Squaler" lifeline rescues for drowning prevention. International Journal of Aquatic Research and Education, 3(3), 315-321.

Petrass, L.A., Blitvich, J.B., McElroy, K., Harvey, J., \& Moran, K. (2012). Can you swim? Self-report and actual competencies among young adults in Ballarat, Australia. International Journal of Aquatic Research and Education, 6(2), $136-148$.

Podstawski, R. (2006). Sprawność fizyczna i opinie na temat profilaktyki zagrożeń dla zdrowia studentów pierwszego roku uniwersytetu Warmińsko-Mazurskiego w Olsztynie w roku akademickim 1999/2000 [Physical ability and opinions on health prevention among first-year students at the University of Warmia \& Mazury in Olsztyn in the academic year of 1999/2000]. Olsztyn: UWM Press.

Podstawski, R., Boraczyńska, S., Skibniewska, K., \& Kolankowska, E. (2013). Can Polish university female students swim? Pedagogics, Psychology, Medical-Biological Problems of Physical Training and Sport, 6, 69-73.

Podstawski, R., \& Choszcz, D. (2016). Weekly, daily and hourly use of the university pool in summer for sportrecreational purposes: Initial research. In J. Sadowski \& T. Niźnikowski (Eds.), Coordination Abilities in Physical Education, Sports and Rehabilitation (pp. 161-173). Biała Podlaska: Józef Piłsudski Academy of Physical Education in Warsaw.

Podstawski, R., Choszcz, D., Klimczak, D., Kolankowska, E., \& Żurek, P. (2014). Habits and attitudes of first-year female students at Warmia \& Mazury University: A call for implementing health education programme at universities. Central Europe Journal of Public Health, 22(4), 143-146.

Podstawski, R., Gizińska, R., \& Kolankowska, E. (2014). The influence of socioeconomic factors on swimming skills of young Polish women aged 19-20. IPEHSS, 3(1), 1-14. 
Podstawski, R., Górnik, K., \& Gizińska, R. (2013). Habits and attitudes of first year students at Warmia \& Maury University, Poland regarding healthy lifestyle. Education in Medicine Journal, 5(3), e64-e76.

Podstawski, R., Klimczak, J., Kolankowska, E., \& Gizińska, R. (2013). The use of Warmia and Mazury Beaches by young women for recreational and health purposes. Polish Journal of Natural Science, 28(1), 107-118.

Podstawski, R., Kolankowska, E., Gizińska, R., Klimczak, J. \& Górnik, K. (2013). Influence of selected environmental factors on the use of beaches in the Warmia and Mazury region of Poland by young women. Polish Journal of Natural Science, 2, 253-266.

Podstawski, R., Mańkowski, S., \& Choszcz, D. (2016). Subjective and objective assessments of swimming and rescue skills in students of the University of Warmia and Mazury in Olsztyn, Poland. In J. Sadowski \& T. Niźnikowski (Eds.), Coordination Abilities in Physical Education, Sports and Rehabilitation (pp. 174-188). Biała Podlaska: Józef Piłsudski Academy of Physical Education in Warsaw.

Rabiej, M. (2012). Statystyka z programem Statistica /Stitistics woth Statistica programme/. Gliwice: Wyd Helion.

Robertson, L.S. (1992).The validity of self-reported behavioural risk factors. Journal of Trauma, 32, 58-59.

Roche, A.F., \& Sun, S.S. (2003). Human Growth: Assessment and Interpretation. Cambridge: Cambridge University Press.

Ross, S.R., Irwin, C.C, Irwin, R.L., Martin, N.Y., \& Ryan, T.D. (2014). The development of swimming skills for African American youth: Parent and caregiver perceptions of barriers and motivations. International Journal of Aquatic Research and Education, 8(3), 219-239.

Schmidt, H-W. (2002). How Europeans Go on Holiday. Statistics in Focus: Industry, Trades and Services. Luxemburg: Eurostat. (Theme $4-15 / 2002$ )

Sport England. (2015). Further decline in swimming numbers dominates latest sports figures. London: Sport England. Retrieved February 21, 2016, from:

https://www.sportengland.org/news-and-features/news/2015/june/11/further-decline-in-swimming-numbers-dominatelatest-sports-figures/

Sport New Zealand. (2015). Sport and active recreation in the lives of New Zealand adults 2013/14. Wellington: Sport New Zealand. Retrieved February 22, 2016, from: http://www.sportnz.org.nz/assets/uploads/attachments/managingsport/research/sport-and-active-recreation-in-the-lives-of-new-zealand-adults.pdf

Sport New Zealand and Auckland Council. (2015). Sport and Active Recreation in the Lives of Auckland Adults: Results from the 2013/14 Active New Zealand Survey. Wellington: Sport New Zealand. Downloaded from: http://www.srknowledge.org.nz/wp-content/uploads/2016/02/2013-14-Regional-Profile-Auckland-FINAL-1.pdf

Stanley, T., \& Moran, K. (in press). Parental perceptions of water competence and drowning risk for themselves and their children in an open water environment. International Journal of Aquatic Research and Education.

Steptoe, A., Wardle, J., Cui, W., Bellisle, F., Zotti, A.M., Baranyai, R., \& Sanderman, R. (2002). Trends in smoking, diet, physical exercise, and attitudes toward health in European university students from 13 countries, 1990-2000. Preventive Medicine, 35(2), 97-104.

US Census Bureau. (2012). Statistical Abstract of the United States: 2012. Arts, Recreation, and Travel: Participation in Selected Sports Activities 2009. Suitland, MD: US Census Bureau.

Waller, S., \& Norwood, D.M. (2011). What African Americans can do to bridge the swimming gap: A Call to Action. International Journal of Aquatic Research and Education, 5(4), 449-475.

AUTHOR'S ADDRESS: $\quad$ Robert Podstawski

University of Warmia \& Mazury in Olsztyn

Department of Physical Education and Sport

Prawocheńskiego 7, 10-720 Olsztyn,

Poland

E-mail: podstawskirobert@gmail.com

Received: 23 September 2016; Accepted: 10 November 2016 